\title{
Crocin-Induced Endothelium-Dependent Relaxation in Isolated Rat
}

\section{Aorta}

\author{
Bibi Marjan Razavi, ${ }^{1}$ Meysam Seydali Seyfabad, ${ }^{1}$ Hossein Hosseinzadeh, ${ }^{2}$ and Mohsen Imenshahidi ${ }^{2,}$ \\ ${ }^{1}$ Department of Pharmacodynamy and Toxicology, School of Pharmacy, Mashhad University of Medical Sciences, Mashhad, Iran \\ ${ }^{2}$ Department of Pharmacodynamy and Toxicology, Pharmaceutical Research Center, School of Pharmacy, Mashhad University of Medical Sciences, Mashhad, Iran \\ "Corresponding author: Mohsen Imenshahidi, Department of Pharmacodynamy and Toxicology, Pharmaceutical Research Center, School of Pharmacy, Mashhad University of \\ Medical Sciences, Mashhad, Iran. Tel: +98-5118823255, Fax: +98-5118823251, E-mail: imenm@mums.ac.ir
}

Received 2015 November 22; Revised 2016 May 29; Accepted 2016 September 14.

\begin{abstract}
Background: Crocin, an active ingredient of saffron, has been found to possess hypotensive effects.

Objectives: The aim of this study was to evaluate the mechanism of vasodilatory effects induced by crocin on isolated rat aorta.

Methods: To study the vasodilatory effects of $\operatorname{crocin}(0.1,0.2,0.4$ and $0.8 \mathrm{M})$, isolated rat thoracic aorta rings were contracted by $10^{-6}$ $\mathrm{M}$ Phenylephrine (PE) or $80 \mathrm{mM}$ of $\mathrm{KCl}$. To assess the role of nitric oxide in mechanism of relaxation, induced by crocin, aortic rings were incubated by L-NG-Nitroarginine methyl ester (L-NAME: $10^{-6} \mathrm{M}$ ) for 20 minutes. Furthermore, the relaxant effect of crocin was evaluated both in intact and denuded endothelium aortic rings.

Results: Crocin induced relaxation in endothelium-intact aortic rings precontracted with PE in a concentration dependent manner yet not in $\mathrm{KCl}$ precontracted aortic rings. The relaxant activity of crocin was abolished by incubation of aortic rings with L-NAME. In addition, the endothelium removal inhibited the relaxant activity of crocin.

Conclusions: This study indicated that relaxant activity of crocin is mainly mediated through an endothelium-dependent mechanism.
\end{abstract}

Keywords: Crocus sativus L., Isolated Rat Aorta, L-NAME, Hypertension

\section{Background}

Crocus sativus L., (C. sativus) commonly known as saffron, is widely cultivated in Iran and other countries (1-4). Crocin is a carotenoid isolated from $C$. sativus and is responsible for the red color of saffron. Crocin is considered as a pharmacologically saffron active component (5). According to several pharmacological studies, crocin can be considered as a new therapeutic agent. Different properties including anticonvulsant $(6,7)$, antidepressant (8-10), anti-inflammatory and antinociceptive $(11,12)$, antioxidant and radical scavenging (13), antitumor $(14,15)$, memory improving $(16,17)$, and hypotensive (18-20) effects have been attributed to crocin. Crocin also showed protective effects on some toxic agents including diazinon and acrylamide in rat brain, as well as liver and cardiovascular system both in in-vitro and in-vivo experiments (21).

Hypertension is one of the prevalent cardiovascular diseases and is an important risk factor for developing other diseases such as metabolic syndrome, endothelial dysfunction, renal dysfunction, diabetes, congestive heart failure, coronary artery disease and stroke (22). Since some adverse effects have been reported following antihypertensive drugs (23), recently natural plants and their active components have been considered as new antihyperten- sive drugs with safety, efficacy, cultural acceptability and lesser side effects (24).

The cardiovascular protective effects of saffron and its active components have been established in several studies. It has been shown that the aqueous-ethanol extract of C. sativus possesses a potent inhibitory effect on heart rate and contractility of guinea pig heart via calcium channelblocking effect (25). Furthermore, the hypotensive effect of $C$. sativus petals extract has been shown in rats (26). In our previous study, the hypotensive effect of intravenous administration of saffron stigma aqueous extract as well as its two major constitutes, crocin and safranal, in normotensive and hypertensive anaesthetized rats, have been reported (18). In another study, chronic administration of crocin reduced the mean systolic blood pressure in Desoxycorticosterone Acetate (DOCA) salt treated rats (27).

\section{Objectives}

Considering the hypotensive effect of crocin, the aim of this study was to find whether crocin shows vasodilatory effects on isolated rat aorta. In addition, the mechanism of vasodilatory effects induced by crocin on isolated rat aorta was evaluated. 


\section{Methods}

\subsection{Plant}

Crocus sativus L. stigma were collected from Ghaen (Khorasan province, northeast Iran) and analyzed in accordance to the ISO/TS 3632-2. Crocin was extracted and purified as defined by Hadizadeh et al. (28).

\subsection{Animal}

Adult male Wistar rats (weight 200 - 250 g) were provided by the animal center of the school of pharmacy, Mashhad University of Medical Sciences. They were kept on a 12-hour light/dark cycle and at a temperature of $23 \pm 1^{\circ} \mathrm{C}$ with free access to food and water. These conditions were maintained constant throughout the experiments. All animal experiments were carried out in accordance to Mashhad University of Medical Sciences, ethical committee Acts.

\subsection{Drugs}

Phenylephrine hydrochloride (PE), acetylcholine chloride (ACh), NG-nitro-L-arginine methyl ester(L-NAME) were purchased from Sigma-Aldrich (Germany). Other chemicals used in this study were provided by Merck (Germany).

\subsection{Tissue Preparation}

Rats were killed by intraperitoneal (IP.) injection of ketamine/xylazine. Then, the thorax was opened and the thoracic aorta was quickly removed and cleaned from adherent connective tissues and cut into rings (4 - $5 \mathrm{~mm}$ in length). Special care was taken to ensure that the endothelial was not damaged during tissue preparation. Two stainless steel stirrups were passed through the lumen of each ring. One stirrup was connected to an isometric force transducer (POWERLAB, AD Instrument, Australia) to measure tension in the vessels. The rings were placed in a 25$\mathrm{mL}$ organ chamber (Organ Bath, 4 channels, AD Instrument, Australia) containing Krebs solution gassed with 95\% $\mathrm{O}_{2} / 5 \% \mathrm{CO}_{2}$, and maintained at $37^{\circ} \mathrm{C}$. The composition of Krebs solution was as follows: $118.0 \mathrm{mM} \mathrm{NaCl}, 4.7 \mathrm{mM}$ $\mathrm{KCl}, 1.2 \mathrm{mM} \mathrm{NaH}_{2} \mathrm{PO}_{4}, 1.2 \mathrm{mM} \mathrm{MgSO}_{4}, 25.0 \mathrm{mM} \mathrm{NaHCO}_{3}, 11.1$ $\mathrm{mM}$ glucose, and $2.5 \mathrm{mM} \mathrm{CaCl}_{2}(\mathrm{pH}=7.4)(29)$. Rings were placed under a resting tension of $2 \mathrm{~g}$ (in preliminary studies determined to be optimum), and allowed to equilibrate for one hour. During this equilibrium period the physiological salt solution was replaced every 15 minutes. The lab chart 7.3 (AD-Instruments) software was used for this study.

\subsection{Vasodilatory Effects of Crocin on the Thoracic Aorta Rings}

To study the vasodilatory effects of crocin, isolated rat thoracic aorta rings were contracted by $10^{-6} \mathrm{M} \mathrm{PE}$ or $\mathrm{KCl} 80$ $\mathrm{mM}$ in two separate experiments, when the vasoconstriction curves of rings reached the plateau phase of the maximum tension, crocin (0.1, 0.2, 0.4 and $0.8 \mathrm{M})$ was added, and the tensions were recorded. The vasodilatory effect of crocin was expressed as a percentage of relaxation to maximum constriction induced by $10^{-6} \mathrm{M} \mathrm{PE}$ or $\mathrm{KCl} 80 \mathrm{mM}$.

The vasodilatory effect of crocin on constriction, induced by PE on rat thoracic aorta, was also performed both on intact endothelium and denuded endothelium rings. The presence of functional endothelium was confirmed by the ability of $\mathrm{ACh}\left(10^{-5} \mathrm{M}\right)$ to induce $\geq 80 \%$ relaxation of rings precontracted with $\mathrm{PE}\left(10^{-6} \mathrm{M}\right)$.

To examine whether crocin-induced vasorelaxation is mediated by the NO, aortic rings were incubated by LNAME $\left(10^{-5} \mathrm{M}\right)$ for 20 minutes. Then, thoracic aortic rings were contracted by $10^{-6} \mathrm{M}$ PE and the vasorelaxant effect of crocin $(0.1,0.2,0.4$ and $0.8 \mathrm{M})$ was then examined.

\subsection{Statistical Analysis}

All results are expressed as mean \pm Standard Error of the Mean (SEM). Analysis of variance (ANOVA) followed by Tukey-Kramer tests were performed to compare means. $\mathrm{P}$ values less than 0.05 were considered significant.

\section{Results}

4.1. Crocin-Induced Vasorelaxation in Endothelium-Intact Aortic Rings Precontracted by PE

As shown in Figure 1, crocin (0.1, 0.2, 0.4 and $0.8 \mathrm{M}$ ) induced relaxation in endothelium-intact aortic rings precontracted with PE $\left(10^{-6} \mathrm{M}\right)$ in a concentration dependent manner. Maximum relaxation response induced by the highest concentration of crocin $(0.8 \mathrm{M})$ was about $50 \%$.

\subsection{Vasodilatory Effect of Crocin in Endothelium-Intact Aortic} Rings Pre-Incubated With L-NAME

Incubation with L-NAME for 20 minutes led to the significant differences in relaxation induced by crocin at different concentrations in endothelium-intact aortic rings precontracted with $\mathrm{PE}\left(10^{-6} \mathrm{M}\right)$ in comparison with relaxation induced by crocin in the absence of L-NAME $(\mathrm{P}<$ 0.001) (Figure 2).

\subsection{Vasodilatory Effect of Crocin in Endothelium Denuded Aor- tic Rings}

Significant differences were observed between relaxation induced by crocin $(0.1,0.2,0.4$ and $0.8 \mathrm{M})$ in endothelium intact and endothelium denuded aortic rings $(\mathrm{P}<$ 0.001) (Figure 3) 
Figure 1. Effect of Crocin on Endothelium-Intact Aortic Rings Precontracted by Phenylephrine $\left(10^{-6} \mathrm{M}\right)$

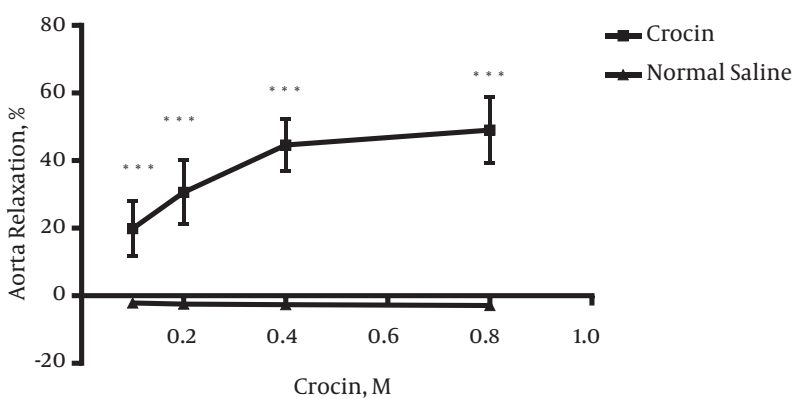

The vasodilatory effect of crocin was expressed as a percentage of relaxation to maximum constriction induced by $10^{-6} \mathrm{M}$ phenylephrine. Values are expressed as mean \pm S.E.M. Saline was used as a control. $\mathrm{P}<0.001$ vs. phenylephrine.

Figure 2. Relaxation Effect of Crocin in Endothelium-Intact Aortic Rings in the Presence of L-NAME $\left(10^{-6} \mathrm{M}\right)$

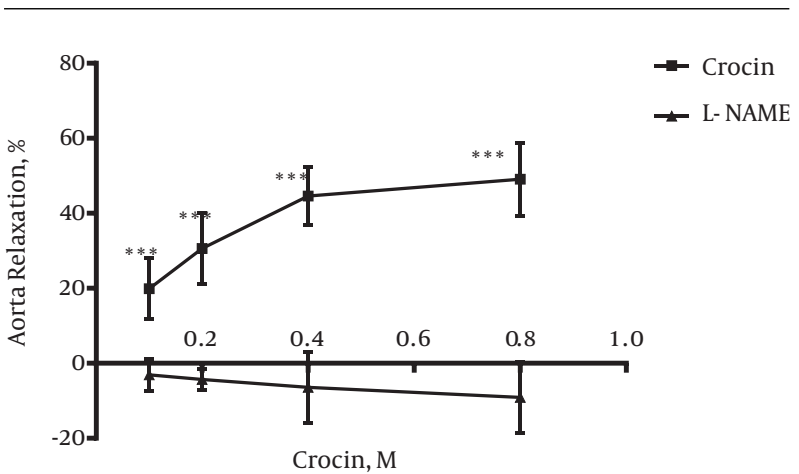

Aortic rings were incubated by L-NAME for 20 minutes. Then, aortic rings were contracted by $10^{-6} \mathrm{M}$ PE and the vasorelaxant effect of crocin was then examined. Values are expressed as mean \pm SEM. ${ }^{* * *} \mathrm{P}<0.001$ vs. L-NAME pre-incubated rings. PE: phenylephrine

Figure 3. Relaxation Effect of Crocin in Endothelium Denuded Aortic Rings Precontracted by phenylephrine $\left(10^{-6} \mathrm{M}\right)$

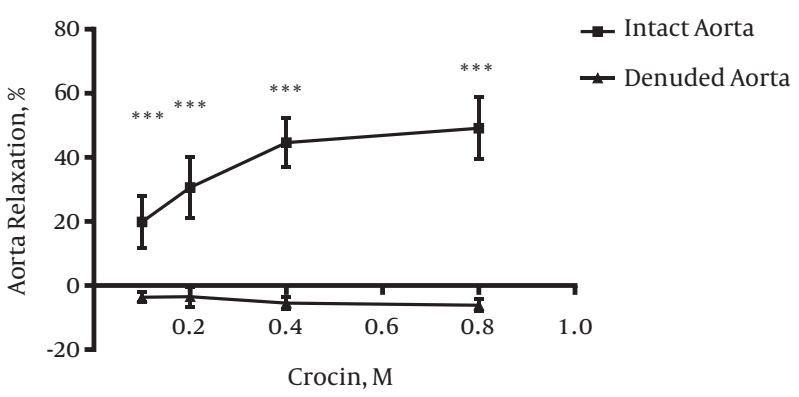

The vasodilatory effect of crocin was expressed as a percentage of relaxation to maximum constriction induced by $10^{-6} \mathrm{M}$ PE both on intact endothelium and denuded endothelium rings. Values are expressed as mean \pm SEM. ${ }^{* * *} \mathrm{P}<0.001$ vs. endothelium intact aortic rings. PE: phenylephrine
4.4. Crocin Could Not Induce Vasorelaxation in EndotheliumIntact Aortic Rings Precontracted by $\mathrm{KCl}$

As shown in Figure 4, crocin could not induce relaxation in endothelium-intact aortic rings precontracted with $\mathrm{KCl}(80 \mathrm{mM})$. Significant differences between relaxation induced by crocin in endothelium-intact aortic rings precontracted by KCl and PE were observed $(\mathrm{P}<0.001)$ (Figure 4).

Figure 4. Effect of Crocin in Endothelium-Intact Aortic Rings Precontracted by $\mathrm{KCl}$ $(80 \mathrm{~m} \mathrm{M})$

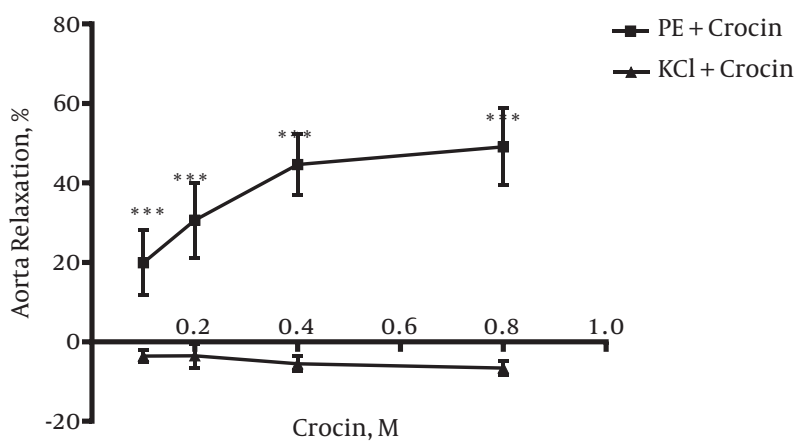

The vasodilatory effect of crocin was expressed as a percentage of relaxation to maximum constriction induced by $\mathrm{KCl}$. Values are expressed as mean \pm S.E.M. ${ }^{* * *} \mathrm{P}<$ 0.001 vs. phenylephrine precontracted rings.

\section{Discussion}

This study revealed that crocin could induce vasorelaxation in isolated rat aorta through an endotheliumdependent mechanism.

According to several investigations crocin is able to reduce blood pressure. Intravenous administration of crocin dose-dependently reduced the mean arterial blood pressure in normotensive and hypertensive anaesthetized rats. For example injection of $200 \mathrm{mg} / \mathrm{kg}$ crocin induced $51 \pm$ $3.8 \mathrm{mmHg}$ reductions in mean arterial blood pressure. The hypotensive effect of crocin in hypertensive animals was more than normotensives. This hypotensive effect did not induce reflex tachycardia (18). Another study revealed that crocin decreased the mean systolic blood pressure in deoxycorticosterone acetate (DOCA) salt-treated rats in chronic administration (27). As crocin caused reduction in blood pressure, in this study the mechanism of crocin hypotensive effect in isolated rat aortic rings was evaluated.

Based on the results, crocin induced vasorelaxation in endothelium intact aortic rings precontracted with PE. To investigate whether the relaxing effect of crocin depended on endothelium-mediated mechanisms, endothelium derived vasodilator relaxing factor such as nitric oxide was 
evaluated $(30,31)$. Nitric oxide is an endothelium releasing factor, which produces by endothelial nitric oxide synthase (eNOS) and induces vascular smooth muscle relaxation through the activation of guanylyl cyclase (32). Nitric oxide has an essential role in the regulation of normal and pathological blood pressure (32).

To evaluate the role of NO in relaxation induced by crocin, the aortic rings were incubated by L-NAME; L-NAME is an inhibitor of nitric oxide synthase. Inhibition of NO by L-NAME inhibited the relaxation induced by crocin. This result showed that NO is involved in crocin relaxant activity.

To verify endothelium-dependent relaxant activity of crocin, another experiment was carried out on endothelium denuded aortic rings. Results indicated that endothelium removal abolished the observed relaxant response in intact endothelium. Therefore, it can be concluded that relaxation effects of crocin is endothelium-dependent. In this study, the effect of crocin on rat isolated aortic rings precontracted by $\mathrm{KCl}$ was also evaluated. It is believed that $\mathrm{KCl}$ depolarizes the cell membrane and increases intracellular calcium via voltage dependent calcium channels of smooth muscle cells and this process leads to contraction whereas, PE (an a1-receptor agonist) activates phosphoinositide signal transduction, and the induced contraction involves receptor-operated calcium and voltage operated calcium channels (33). Results showed that crocin could not induce relaxation in endothelium-intact aortic rings precontracted by $\mathrm{KCl}$, thus, according to this result, it is suggested that L-type calcium channels might not be affected by crocin.

It was reported that crocetin extracted from Gardenia jasminoides improved endothelium-dependent relaxation of aorta isolated from hypercholesterolemic rabbit. The protection induced by crocetin has been attributed to the increased activity of eNOS, which led to the increase of NO production and finally protection against atherosclerosis. It was also indicated that down regulation of eNOS expression as a result of oxidized low density lipoprotein (LDL) and increased NO production in bovine aortic endothelial cells (BAECs) were inhibited by crocetin (34). Result of another study showed that crocin could improve vascular toxic effects of diazinon by restoring altered contractile responses to $\mathrm{PE}$ and $\mathrm{KCl}$ and impaired relaxant responses to acetylcholine and sodium nitroprusside in rat aorta (35). Moreover, crocin restored the effects of sub chronic diazinon administration on systolic blood pressure in rats (36). Taken together, crocin could increase NO production and induce relaxant activity in aortic rings isolated from rats, dose dependently.

In summary, the results of the present study indicated that crocin induced relaxation in isolated rat aortic rings might be due mainly to its effect on endothelium via nitric oxide synthase pathway.

\section{Acknowledgments}

The authors are thankful to the pharmaceutical research center and the vice chancellor of research at the Mashhad University of Medical Sciences for their financial support. The results described in this paper were part of a Pharm. D. thesis.

\section{Footnotes}

Authors' Contribution: Study concept and design: Mohsen Imenshahidi and Hossein Hosseinzadeh.

Funding/Support: This study was supported in part by grant 910473 from the vice chancellor of research, Mashhad University of Medical.

\section{References}

1. Hosseinzadeh H. Saffron: a herbal medicine of third millennium Jundishapur J Nat Pharm Prod. 2014;9(1):1-2. [PubMed: 24644431].

2. Soeda S, Ochiai T, Shimeno H, Saito H, Abe K, Tanaka H. Pharmacological activities of crocin in saffron. J Nat Med. 2007;61:102-11. doi: 10.1007/s11418-006-0120-9.

3. Hosseinzadeh H, Nassiri-Asl M. Avicenna's (Ibn Sina) the Canon of Medicine and saffron (Crocus sativus): a review. Phytother Res. 2013;27(4):475-83. doi:10.1002/ptr.4784. [PubMed: 22815242].

4. Ziaee T, Razavi BM, Hosseinzadeh H. Saffron reduced toxic effects of its constituent, safranal, in acute and subacute toxicities in rats. Jundishapur J Nat Pharm Prod. 2014;9(1):3-8. [PubMed: 24644432].

5. Alavizadeh $\mathrm{SH}$, Hosseinzadeh $\mathrm{H}$. Bioactivity assessment and toxicity of crocin: a comprehensive review. Food Chem Toxicol. 2014;64:65-80. doi: 10.1016/j.fct.2013.11.016. [PubMed: 24275090].

6. Hosseinzadeh $\mathrm{H}$, Khosravan $\mathrm{V}$. Anticonvulsant effects of aqueous and ethanolic extracts of Crocus sativus L. stigmas in mice. Arch Iran Med. 2002:44-7.

7. Hosseinzadeh $\mathrm{H}$, Talebzadeh F. Anticonvulsant evaluation of safranal and crocin from Crocus sativus in mice. Fitoterapia. 2005;76(7-8):7224. doi: 10.1016/j.fitote.2005.07.008. [PubMed: 16253437].

8. Hosseinzadeh H, Karimi G, Niapoor M. Antidepressant effect of Crocus sativus L. stigma extracts and their constituents, crocin and safranal, in Mice. J Med Plants. 2004;3:48-58. doi: 10.17660/actahortic.2004.650.54.

9. Ghasemi T, Abnous K, Vahdati F, Mehri S, Razavi BM, Hosseinzadeh H. Antidepressant effect of Crocus sativus aqueous extract and its effect on CREB, BDNF, and VGF transcript and protein levels in rat hippocampus. Drug Res (Stuttg). 2015;65(7):337-43. doi: 10.1055/s-00341371876. [PubMed: 24696423].

10. Vahdati Hassani F, Naseri V, Razavi B, Mehri S, Abnous K, Hosseinzadeh $\mathrm{H}$. Antidepressant effects of crocin and its effects on transcript and protein levels of CREB, BDNF, and VGF in rat hippocampus. Daru. 2014;8:22. doi: 10.1186/2008-2231-22-16.

11. Hosseinzadeh $\mathrm{H}$, Younesi HM. Antinociceptive and antiinflammatory effects of Crocus sativus L. stigma and petal extracts in mice. BMC Pharmacol. 2002;2:7. [PubMed: 11914135]. 
12. Amin B, Hosseinzadeh H. Evaluation of aqueous and ethanolic extracts of saffron, Crocus sativus L., and its constituents, safranal and crocin in allodynia and hyperalgesia induced by chronic constriction injury model of neuropathic pain in rats. Fitoterapia. 2012;83(5):88895. doi: 10.1016/j.fitote.2012.03.022. [PubMed: 22484092].

13. Hosseinzadeh $\mathrm{H}$, Shamsaie F, Mehri S. Antioxidant activity of aqueous and ethanolic extracts of Crocus sativus L. stigma and its bioactive constituents crocin and safranal. Pharmacogn Mag. 2010;5:419-24.

14. Abdullaev FI. Biological effects of saffron. Biofactors. 1993;4(2):83-6. [PubMed: 8347278].

15. Hosseinzadeh H, Behravan J, Ramezani M, Ajgan K. Anti-tumor and cytotoxic evaluation of Crocus sativus L. stigma and petal extracts using brine shrimp and potato disc assays. J Med Plants. 2005;4:59-65.

16. Hosseinzadeh H, Sadeghnia H, Abbasi Ghaeni F, Motamedshariaty V, Mohajeri S. Effects of Saffron (Crocus sativus L.) and its active constituent, crocin, on recognition and spatial memory after chronic cerebral hypoperfusion in rats. Phytother Res. 2012;10:1002.

17. Hosseinzadeh H, Ziaei T. Effects of Crocus sativus stigma extract and its constituents, crocin and safranal, on intact memory and scopolamine-induced learning deficits in rats performing the Morris water maze task. J Med Plants. 2006;5(19):40-50.

18. Imenshahidi $M$, Hosseinzadeh $H$, Javadpour $Y$. Hypotensive effect of aqueous saffron extract (Crocus sativus L.) and its constituents, safranal and crocin, in normotensive and hypertensive rats. Phytother Res. 2010;24(7):990-4. doi: 10.1002/ptr.3044. [PubMed: 20013822].

19. Imenshahidi M, Razavi BM, Faal A, Gholampoor A, Mousavi SM, Hosseinzadeh $\mathrm{H}$. The effect of chronic administration of safranal on systolic blood pressure in rats. Iran J Pharm Res. 2015;14(2):585-90. [PubMed: 25901167].

20. Imenshahidi M, Razavi BM, Faal A, Gholampoor A, Mousavi SM, Hosseinzadeh $\mathrm{H}$. The effect of chronic administration of saffron (Crocus sativus) stigma aqueous extract on systolic blood pressure in rats. Jundishapur J Nat Pharm Prod. 2013;8(4):175-9. [PubMed: 24624210].

21. Razavi BM, Hosseinzadeh $\mathrm{H}$. Saffron as an antidote or a protective agent against natural or chemical toxicities. Daru. 2015;23:31. doi: 10.1186/s40199-015-0112-y. [PubMed: 25928729].

22. Veeramani C, Al-Numair KS, Chandramohan G, Alsaif MA, Alhamdan AA, Pugalendi KV. Antihypertensive effect of Melothria maderaspatana leaf fractions on DOCA-salt-induced hypertensive rats and identification of compounds by GC-MS analysis. $J$ Nat Med. 2012;66(2):302-10. doi: 10.1007/s11418-011-0590-2. [PubMed: 21964566].

23. Krum H, Pellizzer AM. New and emerging drug treatments for hypertension. Aust Fam Physician. 1998;27(4):235-7. [PubMed: 9581329] 2402.

24. Talha J, Priyanka MAA. Hypertension and herbal plants. Int Res JPharm. 2011;2:26-30.
25. Boskabady MH, Shafei MN, Shakiba A, Sefidi HS. Effect of aqueousethanol extract from Crocus sativus (saffron) on guinea-pig isolated heart. Phytother Res. 2008;22(3):330-4. doi: 10.1002/ptr.2317. [PubMed: 18058985].

26. Fatehi M, Rashidabady T, Hassanabad ZF. Effects of petals extracts of saffron on rat blood pressure and on responses induced by electrical field stimulation in the rat isolated vas veferens and guineapig ileum. J Ethnopharmacol. 2003;84:199-203. doi: 10.1016/S03788741(02)00299-4.

27. Imenshahidi M, Razavi BM, Faal A, Gholampoor A, Mousavi SM, Hosseinzadeh $\mathrm{H}$. Effects of chronic crocin treatment on desoxycorticosterone acetate (doca)-salt hypertensive rats. Iran J Basic Med Sci. 2014;17(1):9-13. [PubMed: 24592301].

28. Hadizadeh F, Mohajeri SA, Seifi M. Extraction and purification of crocin from saffron stigmas employing a simple and efficient crystallization method. Pak J Biol Sci. 2010;13(14):691-8. [PubMed: 21848061].

29. Parsaee H, Imenshahidi M, Fatehi Z. Lovastatin incubation improves acetylcholine-induced relaxation in isolated aortic rings of diabetic rat. Iran J Pharmaceut Res. 2006;3:191-8.

30. Mombouli JV, Vanhoutte PM. Endothelial dysfunction: from physiology to therapy. J Mol Cell Cardiol. 1999;31(1):61-74. doi: 10.1006/jmcc.1998.0844. [PubMed: 10072716].

31. Dehpour AR, Ghafourifar P, Samenian J, Sadeghipour HR, Sadr SS. The effect of lithium on endothelial-dependent relaxation in rat isolated aorta. Gen Pharmacol. 1995;26:1003-7. doi: 10.1016/03063623(94)00286-V.

32. Sausbier M, Schubert R, Voigt V, Hirneiss C, Pfeifer A, Korth M, et al. Mechanisms of NO/cGMP-dependent vasorelaxation. Circ Res. 2000;87(9):825-30. [PubMed: 11055988].

33. Mashhoodi T, Zahedi-Asl S, Sarkaki A. Inhibitory effect of aluminium on $\mathrm{Kcl}$ and phenylephrine induced contraction in isolated rat aorta. Acta Medica Iranica. 2004;42.

34. Tang FT, Qian ZY, Liu PQ, Zheng SG, He SY, Bao LP, et al. Crocetin improves endothelium-dependent relaxation of thoracic aorta in hypercholesterolemic rabbit by increasing eNOS activity. Biochem Pharmacol. 2006;72(5):558-65. doi: 10.1016/j.bcp.2006.05.018. [PubMed: 16876766].

35. Razavi BM, Hosseinzadeh H, Abnous K, Imenshahidi M. Protective effect of crocin on diazinon induced vascular toxicity in subchronic exposure in rat aorta ex-vivo. Drug Chem Toxicol. 2014;37(4):378-83. doi: 10.3109/01480545.2013.866139. [PubMed: 24392635].

36. Razavi M, Hosseinzadeh H, Abnous K, Motamedshariaty VS, Imenshahidi M. Crocin restores hypotensive effect of subchronic administration of diazinon in rats. Iran J Basic Med Sci. 2013;16(1):64-72. [PubMed: 23638294]. 